Supplement of Biogeosciences, 17, 979-993, 2020

https://doi.org/10.5194/bg-17-979-2020-supplement

(c) Author(s) 2020. This work is distributed under

the Creative Commons Attribution 4.0 License.

(c) (1)

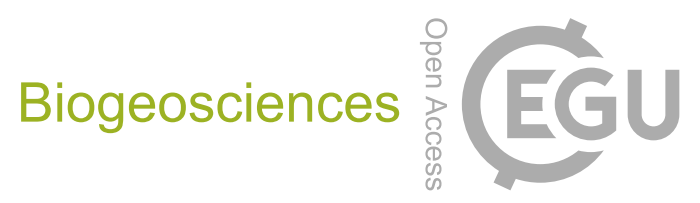

Supplement of

\title{
Isotopic evidence for alteration of nitrous oxide emissions and producing pathways' contribution under nitrifying conditions
}

\section{Guillaume Humbert et al.}

Correspondence to: Guillaume Humbert (g.humbert86@gmail.com)

The copyright of individual parts of the supplement might differ from the CC BY 4.0 License. 


\section{Supplementary material description}

2 Six pages of supplementary material containing two tables and three figures. 


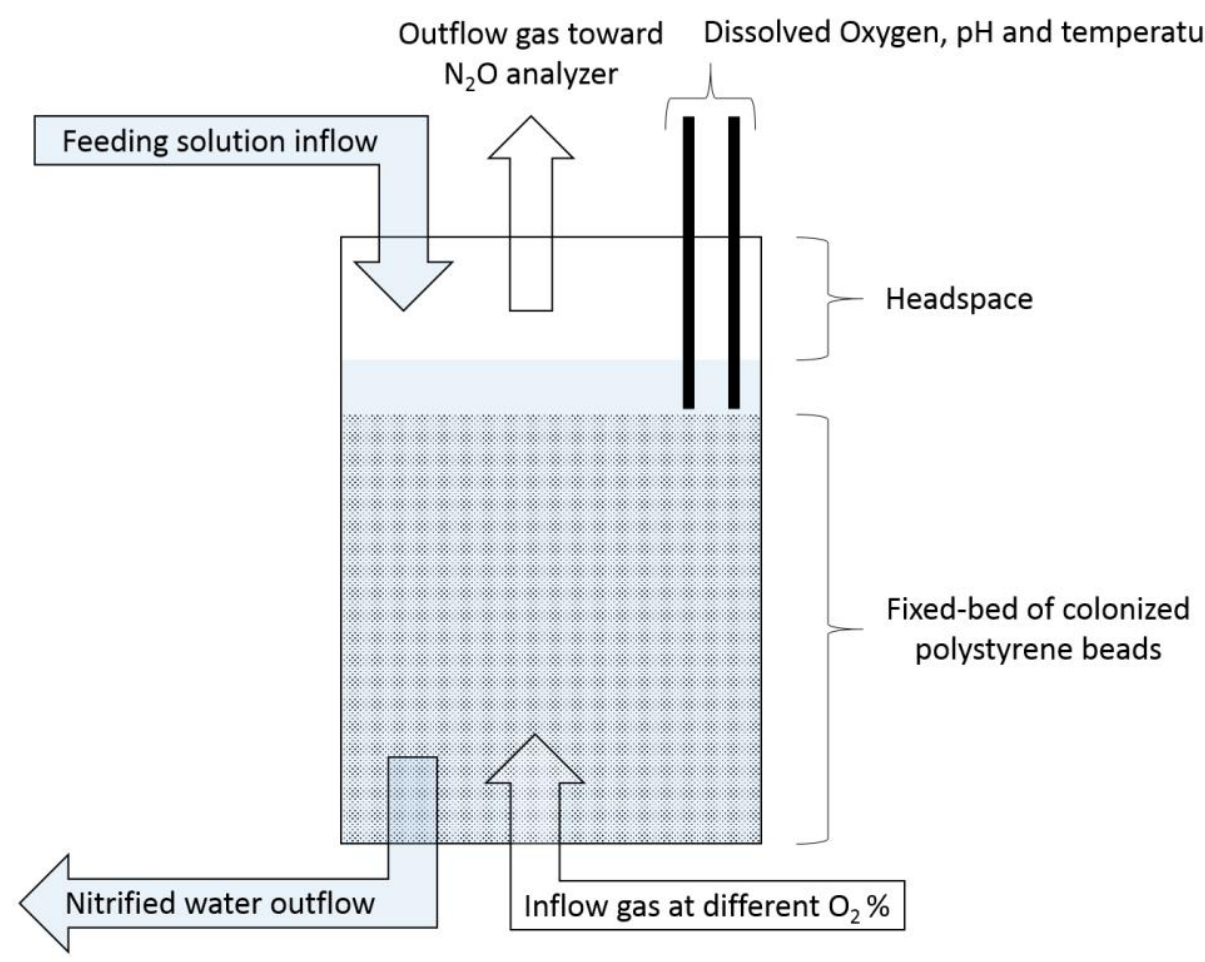

Figure S1. Schematic overview of the nitrifying reactor used in this study. Note that solution was down-flowing, while air was up-flowing. 


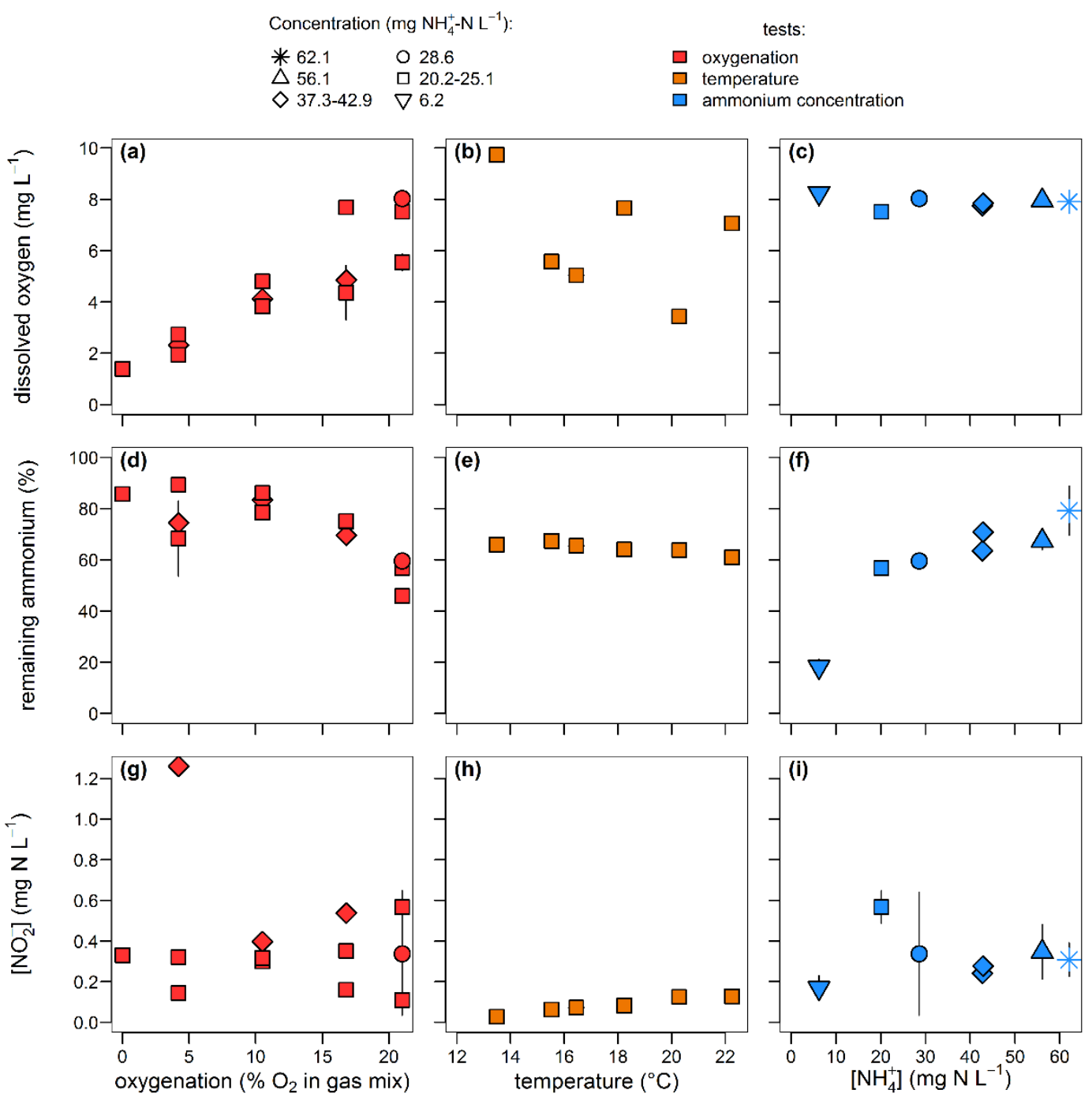

Figure S2. Effect of oxygenation, temperature and ammonium concentration on (a-c) dissolved oxygen concentration, (d-f) the remaining ammonium, and (g-i) the nitrite concentration. 
6 Table S1. Inflow and outflow ammonium, nitrite and nitrate concentrations measured during the ammonium 7 concentration tests.

\begin{tabular}{|c|c|c|c|c|c|}
\hline \multicolumn{2}{|c|}{$\left[\mathbf{N H}_{4}{ }^{+}\right]\left(m g N L^{-1}\right)$} & \multicolumn{2}{|c|}{$\left[\mathbf{N O}_{2}{ }^{-}\right]\left(m g N L^{-1}\right)$} & \multicolumn{2}{|c|}{$\left[\mathbf{N O}^{-}\right]\left(m g N L^{-1}\right)$} \\
\hline inflow & outflow & inflow & outflow & inflow & outflow \\
\hline $6.2 \pm 0.1$ & $1.1 \pm 0.2$ & 0 & $0.2 \pm 0.1$ & 1.8 & $5.1 \pm 0.3$ \\
\hline $20.2 \pm 0.5$ & $11.5 \pm 0.2$ & $0.9 \pm 0.2$ & $0.6 \pm 0.1$ & $3.1 \pm 0.4$ & $9.6 \pm 0.1$ \\
\hline $28.6 \pm 0.5$ & $17 \pm 0.8$ & 0 & $0.3 \pm 0.1$ & 1.4 & $9.3 \pm 0.7$ \\
\hline $42.7 \pm 1.0$ & $27.1 \pm 1.6$ & 1.3 & $0.2 \pm 0.1$ & $4.9 \pm 1.6$ & $14 \pm 0$ \\
\hline 42.9 & 30.4 & 0.1 & 0.3 & 1.4 & 13.5 \\
\hline $56.1 \pm 0.3$ & $37.8 \pm 2$ & $0.1 \pm 0.1$ & $0.3 \pm 0$ & $4.5 \pm 0.1$ & $17.9 \pm 1.4$ \\
\hline $62.1 \pm 0.4$ & $49.1 \pm 5.9$ & 0 & $0.3 \pm 0.3$ & 1.4 & $10 \pm 0.2$ \\
\hline
\end{tabular}




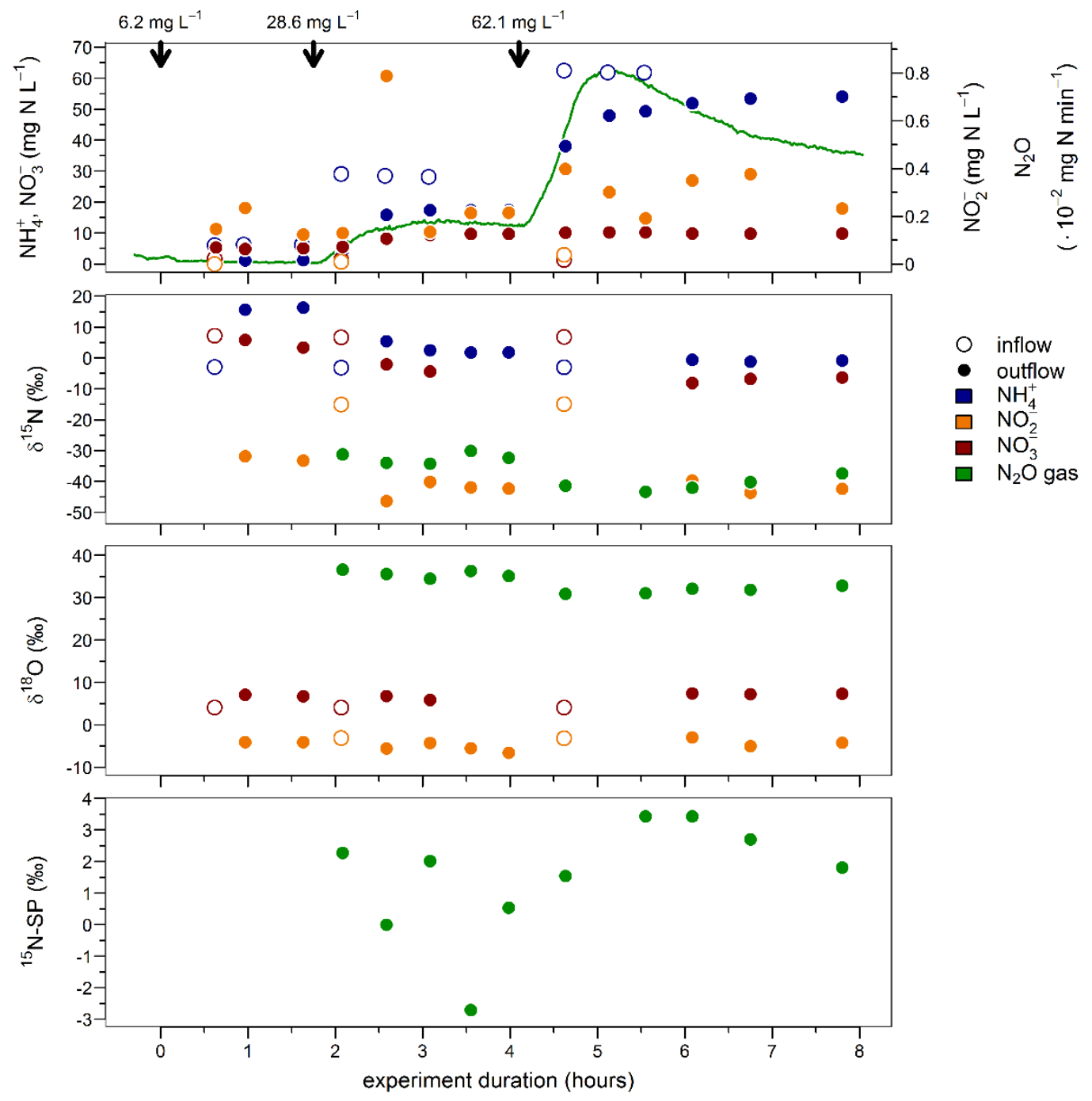

Figure S3. Concentrations, and nitrogen and oxygen isotope ratios of ammonium $\left(\mathrm{NH}_{4}^{+}\right)$, nitrite $\left(\mathrm{NO}_{2}^{-}\right)$, nitrate $\left(\mathrm{NO}_{3}^{-}\right)$ and nitrous oxide $\left(\mathrm{N}_{2} \mathrm{O}\right)$ measured during the increasing ammonium concentration experiment. Nitrogen isotopomer site-preference $\left({ }^{15} \mathrm{~N}-\mathrm{SP}\right)$ was also calculated. 
Table S2. Inflow and outflow ammonium, nitrite and nitrate concentrations and nitrogen isotope ratios measured during the ammonium concentration tests presented in Fig. S3. Average concentration and nitrogen isotope ratio were calculated for the $\mathrm{NO}^{-}$pool (i.e. $\mathrm{NO}_{2}{ }^{-}$and $\mathrm{NO}_{3}{ }^{-}$). Note that the nitrogen isotope ratio of inflow samples was measured only once in each experiment.

\begin{tabular}{|c|c|c|c|c|c|c|c|}
\hline$\left[\mathrm{NH}_{4}{ }^{+}\right]$ & {$\left[\mathrm{NO}_{2}{ }^{-}\right]$} & {$\left[\mathrm{NO}_{3}{ }^{-}\right]$} & \multirow[t]{2}{*}[\mathbf{NO}_{\mathbf{x}}^{-}]{} & $\delta^{15} \mathrm{~N}-\mathrm{NH}_{4}{ }^{+}$ & $\delta^{15} \mathrm{~N}-\mathrm{NO}_{2}^{-}$ & $\delta^{15} \mathrm{~N}-\mathrm{NO}_{3}{ }^{-}$ & $d^{15} N-N O_{x}^{-}$ \\
\hline \multicolumn{3}{|c|}{$m g N l^{-1}$} & & \multicolumn{4}{|c|}{$\% o$} \\
\hline \multicolumn{4}{|c|}{ inflow } & \multicolumn{4}{|c|}{ inflow } \\
\hline 6.08 & 0 & 1.76 & 1.76 & -2.9 & n.a. & 7.2 & 7.2 \\
\hline 29.09 & 0.01 & 1.38 & 1.39 & -3.1 & -15.1 & 6.7 & 6.5 \\
\hline 62.51 & 0.04 & 1.35 & 1.39 & -3 & -14.9 & 6.8 & 6.2 \\
\hline \multicolumn{4}{|c|}{ outflow } & \multicolumn{4}{|c|}{ outflow } \\
\hline $1.22 \pm 0.05$ & $0.18 \pm 0.06$ & $4.95 \pm 0.14$ & 5.13 & $16 \pm 0.4$ & $-32.5 \pm 1$ & $4.6 \pm 1.4$ & 3.3 \\
\hline $17.02 \pm 0.72$ & $0.31 \pm 0.08$ & $9.26 \pm 0.66$ & 9.60 & $2.9 \pm 1.6$ & $-42.7 \pm 2.6$ & $-3.2 \pm 1.4$ & -4.6 \\
\hline $49.14 \pm 5.63$ & $0.34 \pm 0.28$ & $10.01 \pm 0.19$ & 10.32 & $-0.9 \pm 0.3$ & $-41.9 \pm 2$ & $-7.1 \pm 0.8$ & -8.1 \\
\hline
\end{tabular}

